\title{
Body and Movement Visualisations in New Media Dance
}

\author{
Paula Varanda \\ Middlesex University - School of Media and Performing Arts \\ Av. Conde de Valbom, $\mathrm{nr} 2,1^{\circ} \mathrm{dt}^{\circ}$. 1050-068 Lisboa. Portugal \\ paulavaranda.pt@gmail.com
}

\begin{abstract}
The primacy of vision in perception and the critique of disembodiment have been central issues in new media theory and remain troublesome in digital culture discussions. These concerns matter to performance artists and they partially explain why digital technologies are scarcely used to make and show new compositions outside the theatre venue. However, some new media dance artworks exist, which are good examples that counterweigh associations of the digital with the artificial, and fears that the body evaporates in the information network and screen surfaces of virtual reality. Combining Frank Popper's concept of techno-aesthetics with principles of dance analysis, this paper discusses three different projects where body and movement visualizations depend on the technologies and ideas involved to make the artworks. Popper argues that virtual art humanizes computer technologies with its emphasis on interactivity and multisensorial features, reflecting a new philosophical understanding of the virtual. I propose extending his framework, which is aimed at the plastic arts, to analyse works where dance has a principal position. This will highlight how the artists draw on the technique by assimilating the medium, and the artworks humanize technology hence drawing on the philosophical debate.
\end{abstract}

Body. Dance. Performance. New media. Cyberspace. Interactivity. Techno-aesthetics.

\section{INTRODUCTION}

Disembodiment and the primacy of vision are central issues in new media theory since its early stages, indicating that the binary opposition between mind and body that solidified with Modernist thought has not been overcome in contemporary digital culture. In this paper I argue that engaging in the analysis of new media dance performances is a fruitful endeavour that may counterbalance opinions that associate the digital with the artificial and with the disappearance of the body. Because bodily movement is the central medium for the perception of, and communication with, the world these artworks maintain an unequivocal relationship with the real, the organic and the human, even though their visualizations may sometimes reach abstract forms.

In his comprehensive study about digital performance, Steve Dixon recalls that the 'body' issue, which is already controversial in contemporary culture - becomes increasingly complex when its "virtual counterpart" that inhabits digital environments - is under discussion due to the possible transformations that are open to the virtual body (Dixon 2007 p.212). Furthermore, as theories about cyberspace have been disclosing and sometimes criticize, in the realm of data information disembodiment is promoted as an ideal: the mind can travel free from bodily constraints and fictitious identities of the self can be adopted with text or a different body image of a customized avatar. These perspectives perpetuate the split between mind and body that Cartesian theory has defended by opposing the natural to the rational; Dixon considers this opposition to be only challenged by the digital artists that have a performing arts background.

Performance artists are particularly sensitive to the paradigmatic split between body and mind because they expect to bring emotions and sensations through their bodies; they act with their bodies in cyberspace and search for a close harmony and connection between mental creativity and physical dexterity. For Dixon the bodies represented in virtual space always have a relationship with their originals, and those embodied visual images maintain their reference to something material that the audience can relate with (p.215).

My purpose here is to highlight how dance artists for whom diminishing the body means dismissing a 
central element of the art form - contribute to eradicate the mind/body separation paradigm. To do so I will analyse different body representations that visual dancers (or virtual performers) assume and how they act in the virtual environments that three particular artists have created in different projects: a web based dance (cie. Mulleras), an Iphone dance application $(n+n$ Corsino) and a telematic installation (Joseph Hyde). While these works explore the digital as a medium that satisfies some conventional choreographic processes in contemporary dance, hence bringing original approaches to technological tools, they also adapt to the medium and introduce in the art form visualisations and composition methods otherwise unlikely to appear.

Because this practice operates under the principles that Lev Manovich (2001) defines for new media objects, these artworks inherently transpose to territories that transcend the characteristic live sets of performing arts. Therefore, theoretical frameworks that come from other fields of knowledge and focus on other artistic disciplines can add to their contextualization and understanding. In this paper I will take on board the concept of techno-aesthetics developed by Frank Popper to discuss virtual art (2007) and apply his theoretical framework to discuss performative artworks where dance has a central role. By doing so I hope to succeed in demonstrating that these artworks bring fresh and distinctive insights in territories where they do not have a dominant influence on discourse. The need of a two-way flow of dialogue between disciplines and between practice and theory, is at the heart of arguments of performance theories like those of Helen Thomas (in general to dance performance) and Steve Dixon (specifically to digital performance); they support my conviction that we benefit from discussing theoretically what the performance driven artworks bring to new media and virtual art.

I have joined these pieces under the umbrella classification of new media dances because in terms of concept, process and presentation they are deeply connected to the technologies that enable them to be instantiated beyond the physical body and to configure as performative events and open works. The artworks discussed in this essay were also selected because they correspond to three categories that Popper establishes in relation to virtual art: multimedia online works, multimedia and multisensorial offline works, and interactive digital installations.

\section{TECHNO-AESTHETICS}

Frank Popper has focused research on the 20th century history of art that is made with electronic media (see for example Popper 1968 \& 1993). His book From Technological to Virtual Art (2007) provides a comprehensive account for the virtual art works of artists whose institutional references, education and antecedents come from the tradition of the fine arts; Popper highlights the techniques involved in their production and how they relate to contemporary issues that emerge in the era of electronic reproduction, information and communication.

Virtual art is considered by Popper as "a new and refined version of technological art" (2007 p.1) because it is particularly engaged with the humanization of computer technologies, emphasises an interactive relation with the public and explores multisensorial features. Furthermore, virtual art reflects a philosophical understanding of the relationship between the real and the virtual that overcomes the binary dualism that opposed those concepts in previous debates and theorizations. An opposition that, according to Dixon, has restrained the development of dematerialized and duplicated artworks, especially in the domain of performance.

In Benjamin's evaluation of mechanical reproductions, the lack of authenticity is a depreciative factor, which inevitably involves the loss of aura that only an original object or immediate presence contains (Benjamin 1992); this idea extends with Jean Baudrillard's theory of virtual as simulacra, where he criticizes the illusion and fake reality within which we are living, with the increase of time spent in virtual environments (Baudrillard 1994); a similar position comes out of Peggy Phelan's passionate discourse for the preservation of performance as an expression that cannot be reproduced since it relies solely on the real human body and it is otherwise something else that betrays its own ontology (Phelan 1993). Popper's understanding of virtual art is framed by more positive views about the virtual that assume the meaning of potential, which is inherent in the Latin use of the term. In this kind of conceptualization the virtual is something that has the possibility of becoming actual existence regardless of its immaterial nature. Such is the sense implicit in Pierre Lévy's theory, where the virtual connects with openness, diversity and productivity (Lévy 1998).

Virtual art is a technically informed designation because it refers to art made with media that are only available from the 1980s onwards, depending 
directly on the development of digital technologies and Internet communication. However, to these technical circumstances that enable new processes of production and publication, of making and showing, Popper adds that artists working within this area present a clear "techno-aesthetic creative commitment" (2007 p.1) that distinguishes them from other antecedents. Such commitment means that these artists develop particular skills that enable them to use the technologies and explore their creative potential; in doing so they often extend the purpose with which the technologies were designed for in the contexts they have been originated; and they also interpret, through their artworks, issues that are socio-political concerns and that raise epistemological, ontological and ethical questions that emerge from this same and specific technological development.

When making virtual art the artists pursue a double logic, which is "based on the combination of current technical and aesthetic issues" (p.2) and this assists individuals or communities to cope with the flux of information, of people, and identities, the compression of time and space, or the fragmentation and variability that characterize our present.

The aesthetic advances that Popper identifies in virtual art are a consequence of the explorations that appear from the artists' engagement with this particular context of possibilities, which include:

(i) Invading the concrete hardware and the functional and numerical order of computer applications with the subjective human experience, balancing form and code with intimacy and presence in interactive relationships between humans and machines.

(ii) Employing techniques generated by industrial, scientific and military interests in artistic projects, which become political satire that critically readdresses issues like surveillance, censorship, and marketing.

(iii) Embedding the use of these technologies with values of freedom and community, namely by exploring self representation and the notion of public art on the internet, developing open source software and creating platforms for professional networking and audience participation in collective authorship proposals.

While these artists engage in multiple ways with the technologies and introduce innovations in aesthetic research they maintain their ethical commitment of stressing human factors involved in "neocommunicability" - a term Popper uses to refer to the exchange of customized individual information across the globe enabled by Internet communication (2007 p.313-314) - and are aware of their responsibility in operating "in the interests of humankind" (idem p.3).

\section{LAYOUTS FOR VIRTUAL ART}

In the review of current practice that configures his criteria to define virtual art, Popper discusses a large spectrum of work under four different 'layouts'. They correspond to the main traces on which these practices are instantiated and therefore become accessible to the public: materialised digital-based work; multimedia off-line works; interactive digital installations and multimedia on-line works. These categories, which I will now briefly introduce from Popper's perspective, also organize the main concepts that he considers to be informative to the making and the techno-aesthetic value of virtual art.

The materialised digital-based work is the layout that mostly resembles traditional plastic arts. Although using digital processing and methods these works can be identified and distinguished by their substantial form: they configure as prints, objects and films, and can be seen in more conventional exhibition displays. Nonetheless they address technology related issues, namely by rethinking about processes and subjects of representation, as well as cognitive and biological problems. None of the artworks analysed in this article match this kind of configuration.

Off-line works are presented in computer devices and combine different media - sound, pictures, moving image and text - playing with our faculty of relating different sensorial stimulus to make sense of the meaning of a single work. For example, moving graphics provide visualizations of music, and sound can enhance the perception of an image, while intimate touch may counterpoint the artificial predominance of remote control action. In these artworks hypertext and experimental narratives are fairly common strategies to question a habitual relationship with language and linear communication; within the structured range of possibilities provided by the hardware interface and the software programme, the artwork allows individual and creative navigation to the user. I associate with this category the piece Soi-Moi, made for a smartphone by $n+n$ corsino, a company that has substantially researched interactive digital dance compositions that develop in $3 \mathrm{D}$ virtual environments.

Interactive installations increase the importance of full body engagement in the dialogue between visitors, electronic displays and their content, highlighting the significance of immersion. For the visitor to enter, belong to and intervene in the alternative reality provided by the virtual 
environments created by the artists, the sense of physical immersion increases emotional involvement and encourages suspension of disbelief and critical distance. Popper strengthens the importance of "reciprocal aesthetic propositions" (p. 220) since the spectators' autonomous behaviour alters and is welcomed by the virtual proposition that becomes actual once the interaction takes place. Techniques to provide sensorial immersion and clarification of the freedom given to the visitor as well as the rules within which he or she can integrate the installation are crucial for these interactions to be successfully rewarding and mutually influential. The example I propose looking at in this area is the interactive installation Me and My Shadow by Joseph Hyde, where visitors from different countries can access a common virtual space to communicate through movement with each other.

Multimedia online works are always dependent on Internet connection and become actual when the user enters the website. Net art is, according to Popper's analysis upon a considerable number of examples, a category of virtual art strongly marked by the issue of social communication providing an unforeseen 'platform' for encounters between the personal and the public. While the web is controlled by corporative protocols that ensure access, storage and maintenance of information and system, it is equally an 'open space' at least within European and North American countries, where one can "broadcast oneself", and provides showing opportunities for arts that can extend outreach to audiences otherwise only attained by mass media communication. In this case my analysis focuses on a web-based dance called 96 details from cie. Mulleras, a company that became internationally acclaimed for exploring the web as an open studio to contemporary dance.

\section{TECHNO-AESTHETICS IN DANCE ANALYSIS}

Popper applies techno-aesthetics theory to artworks instantiated as materialised objects, as the content of a device, with an installation set up or somewhere in the private/public cyberspace. Such flexibility makes this conceptual framework a suitable tool to analyze dance artworks and observe how their body and movement visualizations specifically relate to the humanist and critical commitment that Popper sees in artists who make virtual art. The techno-aesthetic value of one practice may, nevertheless, change according to sensibilities and concerns that are paramount for some artistic areas but not in others. For example, issues that are revolutionary for artists whose primary reference is a visual tradition based in fixed materials may be elementary principles for the performing arts and vice-versa.
The interplay between the multisensorial and the multimedia is commonplace grammar for the systemic and interdisciplinary constitution of a dance performance, where the body (or bodies,) the movement (and the style, the genre or the dramaturgy), and the visual or aural elements continuously interconnect in a dynamic temporal spatial relationship to create a specific artwork and event. On the other hand, the condition of having the human body as both source and display is critical to the ontology of unmediated dance performance, despite occurring inside a theatre or in a site-specific environment. Hence dance artworks that rely on digital technologies to become public performance can only be positively discussed with the new philosophical understanding of the virtual, which Popper has acknowledged in his definition of virtual art.

I have defended before that an interdisciplinary approach can more accurately address new media dances and their double nature as a digital and physical expression. However, when the concept of techno-aesthetics is employed to analyze artworks from the perspective of a dance studies researcher, specific enquiries that this field tends to launch upon its subjects must also be considered. In her sociologic approach Helen Thomas contends that although dance performance is a cultural product that reflects traditions and beliefs, it is hardly considered a subject in cultural studies. She gives the example of theories on club culture, which privilege considering contextual conditions and scarcely investigate in a descriptive way the behaviour of the dancing bodies (Thomas $1995 \mathrm{p}$. 3 ). As a method for analysis Thomas defends the combination of an extrinsic approach - that examines determining social context where the artwork is made - with an intrinsic approach that examines specific qualities of the dance itself, highlighting components and aesthetic choices that produce the different discourses and styles a dance can embody.

The focus on movement behaviour in dance analysis is coupled with attention to how the body is represented and positioned. Is representation achieved with text, voice or graphics, or with anthropomorphic figures? How many performers feature the work and what are they wearing? Are gender roles visible? And where does the dance happen? An important concern for dance artists is ethical consideration about the relationship between the user/spectator and the performer: is there any power issue involved when one manipulates the other? These aspects are relevant for interpretation of conceptual ideas and extra artistic issues; but they equally inform the understanding of technical options and constraints that influence body representations, movement 
visualizations and choreographic processes that constitute the work, which speak for the artists' techno-aesthetic commitment towards the humanization of information technologies. This methodological approach is undertaken in the following analysis, which takes in consideration my personal experience with the artworks in their original presentation form, and is assisted by informal conversations that I have carried out with the artists.

\section{96 DETAILS - VISUALISATION 1}

French group Cie. Mulleras, has invested remarkably in using the web as a site for new media dance. 96 details (2006-2009) is an on-line work that results from several fragments (96 films of body movement details), which can be combined in sections where small game-like puzzles take form. The company wanted to develop the idea of a living picture where the body could be a designer or a tool to design lines, spaces and patterns, exploring the calligraphic possibilities of choreography. The work has no narrative though, its central thematic concern is about body memories and what is left as a trace of presence, of being human; this aim merges then with the focus on detail and how things that appear to be insignificant may turn into rather important triggers of a more noticeable and consequent action. Didier and Magali Mulleras have set up a cube as the referential shape from which movement develops in space and the several units can be combined; these combinations are potentially possible but only become actual with the user's unique and creative interaction with the work.

Once we enter the www.mulleras.com website and click the piece title, a spinning cube unfolds in six squares, each having three or four options of different 'micro-dances'. Many of them are developed from a single phrase, performed by a woman dressed in white, who moves inside the cubic area, which is smaller than the length of her stretched body. She rolls on her knees, opens her arms, closes her legs, turns the torso, sits or lies down, looks towards the viewer or around herself and pushes her limbs against the limits of the performing space in a smooth, softly paced and continuous choreography. The space is neutral, empty, filled by the body, and the sequence has been filmed from two different camera angles: a top one that strictly confines the body inside a square frame and against a white floor, and a front one that allows the perception of the contour of her movement against the black void of the surrounding space. In each angle the authors used long or close up shot sizes and sometimes the sequence was filmed with different lighting. Post- production effects also introduced visual nuances such as blurring, solarising or contrasting of the image. Finally, several of the available sections of 96 details present duplicates of the same film or scene, allowing one layer of choreographic intervention that creates patterns and abstract drawings from the multiplication of units of pure body movement. All these options have an effect on the many possible variations of 96 details as an artwork; they influence the choreographic score, the body representation, the emotional tension and the overall composition that results from the coincidence and arrangement of these ingredients.

After selecting which micro event to see, the interactive spectator decides on their development by clicking on buttons that change direction, size, colour, speed and juxtaposition of the films and other options regarding sound. Apart from the preset elements available as media fragments that only last a few minutes, the duration of the micro events and the whole experience depends on the choice of the visitor who enters the website; because the system's design is based in a looping operation, the show will go until the user leaves the 'auditorium'.

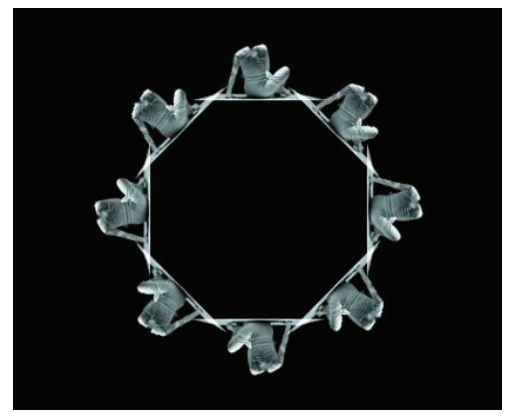

Figure 1: 96 details, Compagnie Mulleras

An important characteristic of 96 details is that the dancing body source material is a collection of samples of two-dimensional photographic moving images. It is only inside the computer system, that this quite raw material is heavily manipulated either by the artists that cut, join, colour and speed the images before display, or by the visiting guests that trigger the sequences and explore various combinations; hence the images of almost abstract and apparently synthetic patterns and traces achieved from multiplication, distortion and repetition, which are key functions in the project, have a very strong sensual quality. While at various times the body loses character identity (we don't see who is there), the movement rhythms and the body-based material originate a sense of intimacy and presence, which enriches the private encounter that is taking place between the audience and the artwork. Another aspect that distinguishes the Mulleras' proposal is that this is a free encounter, which can take place across the 
globe at any time. Although that is relatively natural in relation to the Internet medium, it deliberately undermines the ruling status of theatrical dance performances, which are valued upon notions of immediacy and evanescence and may be commercialized commodities.

\section{SOI MOI - VISUALIZATION 2}

$n+n$ corsino is a French company founded in the 1980s. After an early stage performance phase, followed by a dance films period in the 1990s, the directors Nicole Corsino and Norbert Corsino shifted their focus to work with installation set ups, using motion capture technology to import movement of dance performers and implement choreography in 3D environments. The company has explored various types of displays to present contemporary dance in spaces and architectures other than the cinema screen or the theatre stage.

Soi Moi (2010) is an interactive dance artwork accessible in the form of an application for I-phone that can be played with after download from the Apple iTunes store. The artists wanted to enable an experience with this everyday utility that stimulates sensorial awareness of the user's body when he or she enters into a dialogue with the piece. Technically, once the application is loaded in the smartphone a menu appears with several buttons (with the shape of black stones floating on the screen), each leading to a segment that explores how the various manipulation modalities and features of the device interact with and influence the course of a two minutes dance sequence inserted in fictional scenarios inspired by natural landscapes or real pictures.

The only dancer is a slim woman wearing a grey top and tight black shorts that performs a twominute dance sequence each time one of the menu options is chosen. The body always appears in full size but in a small scale in relation to the environment where the choreography develops. She sits, she walks, she jumps, she twists, she taps, she floats or she falls in landscapes that can be altered with the user's actions, such as shaking, blowing, touching and rotating the frame. In one of the segments the user can take a picture of his/her real environment, which instantly becomes the background of the performance. And in another section the performer blurs the scenery with a trace of her movement. Although all the scenes have their own predetermined sound score, in some cases a tune can be chosen from the user's own music library to accompany the dance.

For these choreographers, working with motion capture and animation technologies was the way to accomplish their interest and commitment in materializing dance in new spaces, where the performers could navigate in imaginary universes and the scale of the human being could be transformed to assume the size of insects and change the human position in relation to nature. On the other hand motion capture was also a significant process to transport real movement signatures of unique performers to these virtual environments instead of synthetic bodies without organic personality. That is one of the reasons why in Soi Moi, although the work is digital and therefore has the potential to take many forms in postproduction or interactivity with the spectator, the gender of the real dancer is the same as the virtual performer, the costumes are very neutral and the source choreography is pre-determined before its last data import.

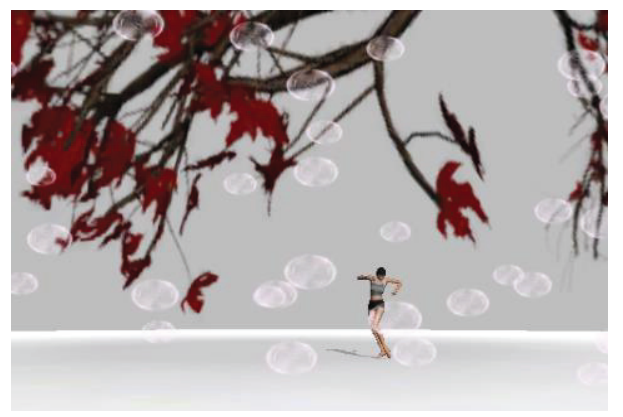

Figure 2: Soi Moi, $n+n$ corsino

This portable installation enables an intense poetic experience offered by a gentle and elegant female that performs together with her partners: the aural and visual environment and the user of the utility. Having the dance piece played, and played with, in our hands approaches the art object and the audience in a particular way, which is very different from having to leave the private home and going to a public venue. It becomes an intimate and creative exchange between the two participating persons that attenuates the separation between human body and machine. The multisensorial constitution of the piece is essential to augment self-awareness and perception of the user's own body, enabling a somatic experience of aesthetic content.

There are artistic reasons related to the fact that the user cannot change the performer's dance sequence, which once initiated cannot be interrupted. This option though, also derives from a conscious ethic position concerning the limits that digital technologies and scientific intervention should have upon the individual and the body behaviour, and they reflect the necessary boundaries of real social interaction that respects the other and aims for a dialectic relationship.

\section{ME AND MY SHADOW - VISUALIZATION 3}

Me and My Shadow (2012) is a project directed by composer and media artist Joseph Hyde, which explores creative interactivity and remote 
presence, enabled by Internet communication and digital data manipulation. This is first and foremost an installation, where visitors generate the content of an improvised performance; they participate in an international telepresence experience and social encounter that occurs on a shared online environment, connected through four separate portals, located in public venues in London, Paris, Istanbul and Brussels.

The new virtual space is a round surface with white floor, similar to the planet seen from the stratosphere, surrounded by a black void where the moon and a few stars mark the sky. Depending on the country from where they enter this performance, the participants' avatars are identified by the colours green, purple, orange and blue.

Although I analyze it from a dance perspective, this project escapes single disciplinary categorization, since visual image and effects, sound, and movement or gestural behaviour are deeply involved and reliant on each other to create the final result. Furthermore the work favours spontaneous and heterogeneous participation rather than specialized skills and has no predetermined content. Nonetheless body and movement are essential defining elements for the artwork to come into being.

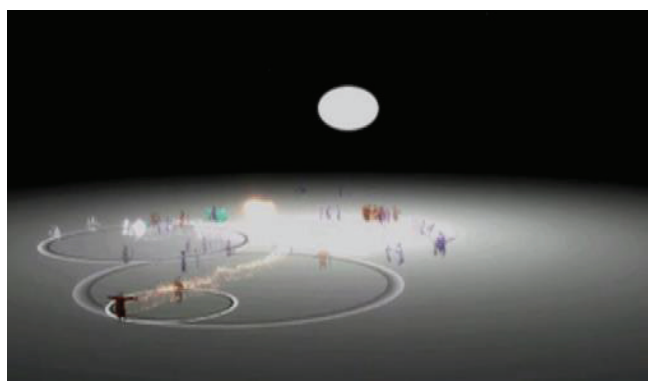

Figure 3: Me and My Shadow, Joseph Hyde

Teleportation and real-time interaction is possible due to Microsoft kinetic cameras that send the 3D movement data of each visitor to be processed inside a complex computer system, which then feeds the live stream back to each portal. Inside the portal the individual visitor's body image is reproduced in real size on a video projection, where the body images of the other visitors from remote locations also appear and meet in the virtual fifth space. The body is represented with transparent and glittering silhouettes and by moving, each visitor can make his/her own avatar take action; navigation in that space allows panning the point of view, progressing towards the centre 'stage' or sliding to the periphery. This performing action also triggers sounds and other shadows of more human bodies, recorded from the movement of previous visitors or the actual ones.
The performers' movement across the space leaves fluctuant trace behind, which enables visualization of the spatial choreographic design.

In Me and My Shadow the body is at the core of human-computer interaction and human-human interaction that is mediated by communication technologies. The dark and concealed box of the portal provides a comfortable place to experiment with gestures and postures that are translated in a real time pleasant feedback of visual and aural effects, which stimulates the visitors to move and become aware of the work through the extension of their own movement; therefore body language is the primary source of expression to 'exist' in the fictional space and communicate with other people that they don't know but might encounter there. Since the body is represented without the usual marks of individuality like face, skin, voice or clothes, the movement becomes central for identity and distinctiveness; people explore dancing skills and exchange utterances with aesthetic value rather than literal meaning. The visitors may feel illiterate as well, and therefore understand that knowledge is also related to the body.

This new system designed with an artistic intent to enable 'digital mobility' introduces social public space as a site for creative and plural collaboration, rehearsing participation and responsibility in the final audiovisual collective outcome. Because the visitors must imply their own bodies, they become performers rather than users. That condition, which is guides the concept of the computer program, the visual design and the interactive system, significantly challenges conventions of mainstream society regarding standardized behaviour in public space, the oneway flow of mass media information and the dominance of word-based language.

\section{CONCLUSIONS}

In the artworks that were here presented, various aspects that Popper considers distinctive in virtual art become evident. They demonstrate how the artists approach the technological and aesthetic context of digital culture and contaminate the codes, machines and telecommunication with human subjectivity. Now that the achievements of these artworks have been introduced separately, some common characteristics will be highlighted.

Although performance is improvised in Me and My Shadow and pre-determined in 96 details and Soi Moi, the three works concentrate on the experience of a solo character, a single person. This choice may be related with technical constraints that affect production since it is easier to collect cleaner and lighter data with a single body, which in turn facilitates postproduction and complexity of the responsive system; digital 
representations of moving human bodies are technically demanding and can look fairly uncanny and artificial when compared with real bodies. If the dance is made by an awkward puppet, and not by a skilful performer, the emotional involvement will changes the difference may jeopardise the whole intended experience. Solo performance is also a significant choice that embodies the problem of individualism and solitude, which is related with the increase of versatile and inexpensive communication media that are accessible in contemporary society. Reinforcing this idea, the performers in Soi Moi and 96 details interact with a solo user who is invited to an intimate exchange that eschews isolation; similarly the solo visitor/user/performer of $M e$ and $M y$ Shadow is invited to be present, with his/her body that carries identity, in remote public space.

Distinguishing these artworks by the technical categories that Popper adopts to analyse virtual art is useful to identify features that they explore and reinvent. However, whether in the conventional web page screen display, the smartphone at the palm of our hand or the room where the whole body moves, interactivity is a formative condition of the three pieces. Again, this condition influences the process of using the hardware, programming the software and conceiving the content, but it equally expresses an ethic position towards the relationship between artwork and audience. These works are open to external participation that reciprocates with new aesthetic propositions; they welcome a non-specialized but nonetheless creative partaking to appear in the moment that the virtual artwork becomes actual and translates in real experience; hence the artists desire the audience's subjective action on which their artworks concretely depend in order to exist.

Finally, the technologies of reproduction, information and communication, which are presently ubiquitous to assist everyday life or the entertainment industry, have been used with a different purpose. These technologies become facilitators of what Dixon calls digital performance in 'cybertheatre' (2007) and their initial utilitarian functions are transcended. The artists have thoughtfully reinstated the human body's protagonist role to generate content and extraordinary movement visualizations.
Rather than seeking alienation, this combination encourages immersion in virtual environments that preserve a connection with the source reality; from that familiarity they may then lead to autonomous organic fictions, giving place to ephemeral encounters with the imaginary and the unspeakable. In this process of poetic and playful experience, body and mind are intensely connected and present.

\section{REFERENCES}

Baudrillard, J. (1994) Simulacra and Simulation. University of Michigan Press, Ann Arbor, Michigan.

Benjamin, W. (1992) Illuminations. Fontana, London.

Dixon, S. (2007) Digital Performance: A History of New Media in Theater, Dance, Performance Art, and Installation. MIT Press, Cambridge, Massachusetts.

Lévy, P. (1998) Becoming Virtual: Reality in the Digital Age. Plenum, New York, London.

Manovich, L. (2001) The Language of New Media, MIT Press, Cambridge, Massachusetts.

Phelan, P. (1993) Unmarked: The Politics of Performance. Routledge, London.

Popper, F. (2007) From Technological to Virtual Art. MIT Press, Cambridge, Massachusetts.

Popper, F. (1993) Art of the electronic age. Thames \& Hudson, London.

Popper, F. (1968) Origins and development of kinetic art. Studio Vista, London.

Thomas, H. (1995) Dance, Modernity and Culture: Explorations in the Sociology of Dance. Routledge London.

\section{WORKS CITED / INTERNET REFERENCES}

Corsino, N. \& N. (2009) Soi Moi, http://www.nncorsino.com/fr/soi-moi/navigation

(video excerpt accessed online 2 March 2013; the piece was visited and played with in March 2012 at the Centre Des Arts, in Enghien-Les-Bains, France)

Hyde, J. (2012) Me and My Shadow http://madeshadow.wordpress.com/about/

(video excerpt accessed 4 Abril 2013; the piece was visited in June 2012 at the National Theatre, London, United Kingdom).

Mulleras, D. \& M. (2006-2009) 96 Details, http://www.mulleras.com/96d/e accueil96d.html (accessed 4 April 2013) 